\title{
CRISPR/Cas9-based genome engineering in HIV gene therapy
}

\author{
Xuanting Tang ${ }^{1}$ \\ ${ }^{1} 140$ Commonwealth Avenue, Boston College, MA, 20467
}

\begin{abstract}
In recent years, the clustered regularly interspaced short palindromic repeats (CRISPR)/CRISPR-associated nuclease (Cas) technology has become the most heated genome editing technique. Comparing to earlier genetic engineering methods, the CRISPR/Cas system is more advantageous due to its simple convenient design, high efficiency, cost-effectiveness, and the ability to perform multi-sites editing simultaneously. As the most effective gene editing tool, it utilizes a simple short RNA-guided mechanism to direct Cas-mediated DNA cleavage at the target genome locus and exploits the endogenous DNA repair pathways to achieve site-specific gene modifications. Initially discovered as a part of the bacterial adaptive immune system, the CRISPR/Cas system has now been widely used to study a broad range of biological genomes. Besides its contribution to our understanding of the basic genetic science, the application of the CRISPR/Cas system also expands rapidly into the medical fields, showing great potentials in the research of genetic diseases, viral infectious diseases, and cancers. In this review, the latest research progress of CRISPR/Cas technology is summarized based on its development, mechanism, and application in HIV/AIDS intervention. This review also examines the existing weaknesses and the future prospects of this promising technology.
\end{abstract}

\section{Introduction}

There has been a persistent search for genome engineering technologies with improved site-specificity, efficiency, and diversity. Perturbations on genomic elements enable researchers to investigate specific gene functions and their associated disease phenotypes on various model organisms ${ }^{1}$. The ability to produce precise alteration to specific DNA sequences in eukaryotic and particularly mammalian cells has significant implications in the fields of biology, medicine, and biotechnology ${ }^{1,2}$. In recent years, various nuclease-based genome editing techniques have been developed to stimulate efficient genome alterations by inducing site-specific double-strand breaks (DSBs) ${ }^{3,4}$. Research studies have demonstrated that the introduction of DSB can stimulate locus-specific gene repairs through either homology-directed recombination (HDR) or non-homologous end joining (NHEJ) ${ }^{3,5-7}$. In the presence of an exogenous homologous repair template, HDR produces precise gene modification at the specific locus with minimal mutagenic activity ${ }^{6-8}$. Alternatively, in the absence of a donor template, DSB ends can be repaired through the error-prone NHEJ mechanism, creating frameshift mutations, including insertion and deletion (indel), at the target site that disrupt normal gene functions ${ }^{6,8,9}$. Overall, DSBs can engender both mutagenesis and gene replacement at the localized break site . $^{5}$.

Three major designer nucleases to be discussed in this review are the zing-finder nuclease (ZFN), the transcription activator-like effector nuclease (TALEN), and the RNA-guided Cas9 endonuclease derived from the CRISPR (clustered regularly interspaced short palindromic repeats) system in the microbial adaptive immune system. Early gene editing technologies use zinc-finger nuclease (ZFN) and transcription activator-like effector nuclease (TALEN) to modify specific DNA sequences. $\mathrm{ZFN}$ is a fusion protein consisted of a nuclease domain of FokI restriction enzyme fused with a series of DNA binding domain ${ }^{10}$. Each zinc finger protein (ZFP) serves as one DNA binding domain that selectively recognizes 3 base pairs (bps) of DNA sequence through protein-DNA interactions ${ }^{5,11}$. Dimerization of two FokI nuclease domains occurs upon $\mathrm{ZFN}$ binding to the recognition sequence, leading to an overall increase of its gene editing specificity ${ }^{12}$ and the formation of DSB at the target genome locus. TALEN is another nuclease-based DNA targeting system with a similar repair mechanism as $\mathrm{ZFN}^{10}$. TALEN is artificially assembled by fusing a series of TALE protein, that each recognizes $1 \mathrm{bp}$ of DNA sequence, to the FokI nuclease ${ }^{13}$. TALEN-mediated cleavage at the specific genome loci can be realized via both in vivo and in vitro methods ${ }^{13,14}$. However, for both strategies, the major challenges are the off-target rate ${ }^{15}$ and the context-dependent binding specificity ${ }^{16,17}$ due to crosstalk between assembled modules within a larger system. Given the difficulties associated with the design, synthesis, and assembly of the DNA-binding domain, researchers have long pursued a more versatile and viable technique for gene editing and regulation.

\footnotetext{
Tangxu@bc.edu
} 


\section{Discovery of CRISPR/Cas system}

CRISPR (clustered regularly interspaced short palindromic repeats)/Cas (CRISPR associated) system was first discovered in E. coli in $1987^{18}$. Over the next decade, interspaced repeat elements with high sequence similarity were found in more than $40 \%$ bacterial genome and most archaeal genome ${ }^{19}$. The CRISPR locus in microbial genomes typically contains a series of well-conserved CRISPR-associated (Cas) genes that appear in conjunction with the signature CRISPR array $^{20,21}$. The CRISPR/Cas system functions in a way that establishes microbial adaptive immune response against foreign genetic materials ${ }^{22-24}$. Unlike ZFN and
TALEN that rely on protein-guided DNA recognition, the CRISPR/Cas system utilizes a simpler RNA-guided mechanism ${ }^{25}$ to produce locus-specific DNA cleavage. By allowing site-specific perturbation at individual genetic elements via base pairing complementarity, the CRISPR/Cas system demonstrates greater precision and efficiency than previous editing technologies ${ }^{26}$. It has developed rapidly and is widely used in research on microbiology, plants, animal science, and various forms of human disease. This review incorporates the development and the principle mechanisms of RNA-guided CRISPR/Cas model, as well as its application in treating viral infectious disease particularly.

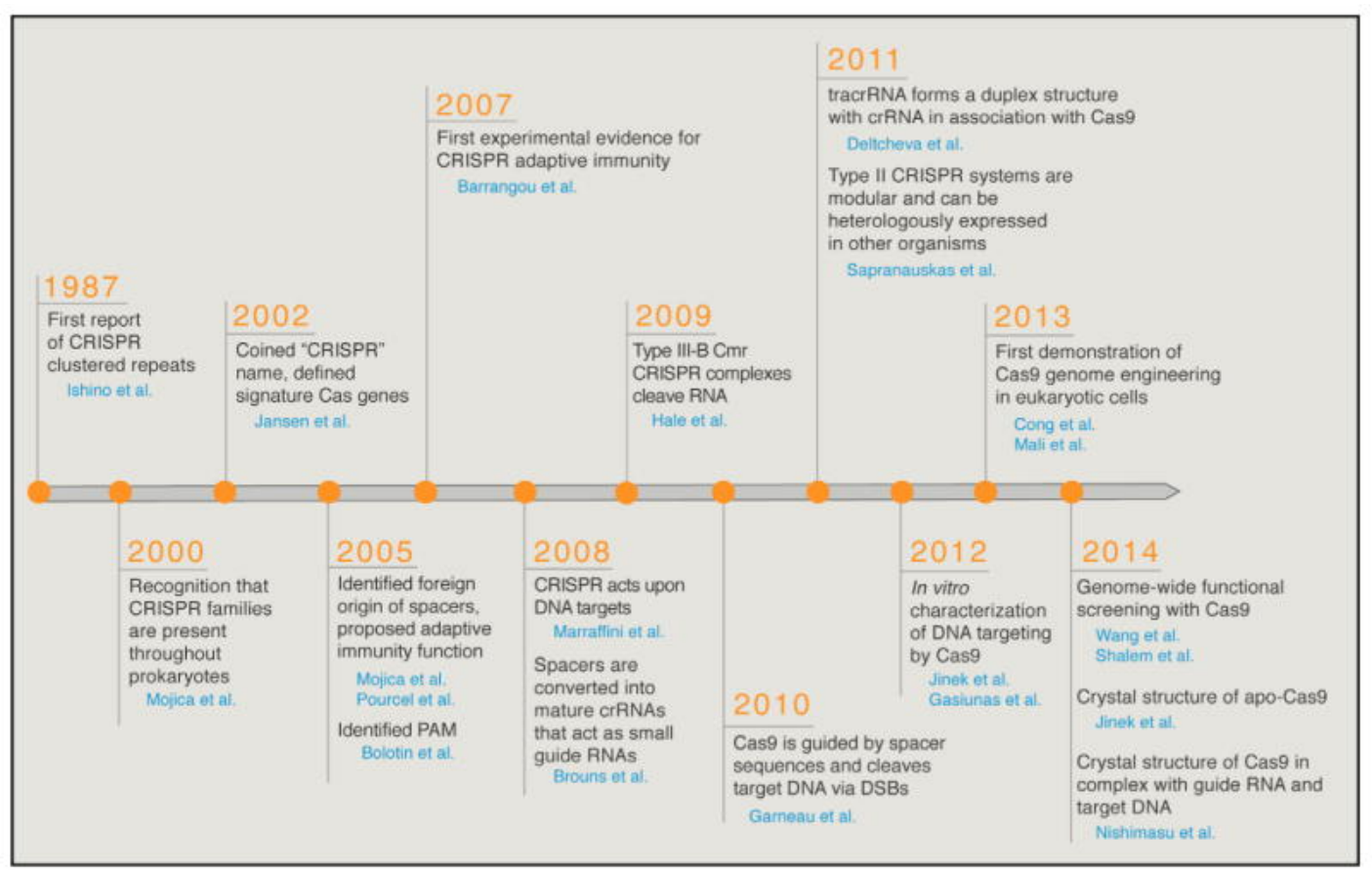

Figure 1: Important Research Studies in the Discovery, Characterization, and Engineering of CRISPR/Cas System. CRISPR (clustered regularly interspaced short palindromic repeats)/Cas (CRISPR associated). DSB (double stranded break). crRNA (CRISPR RNA). tracrRNA (trans-activating CRISPR RNA). ${ }^{5}$

\section{Mechanism of RNA-guided Genome Editing via CRISPR/Cas9}

The CRISPR/Cas system assists prokaryotes to acquire adaptive immunity to foreign genetic material from virus or phage. This approach relies on Watson-Crick base pairing to direct recognition and degradation of specific nucleic acid sequences ${ }^{25,27}$ (Fig. 2). The first process upon the invasion of foreign genetic elements is termed adaptation, whereby a sequence snippet is captured by the Cas1-Cas2 complex ${ }^{28}$ and then integrated into the genomic CRISPR array. The CRISPR array along with the adjacent Cas genes and trans-activating CRISPR RNA (tracrRNA) gene ${ }^{22,29}$ will be expressed together, producing CRISPR RNA (crRNA), Cas effector nuclease, and tracrRNA respectively. For type II CRISPR/Cas system, mature crRNA hybridizes with tracrRNA via complementation $^{30}$; the resulting single-guide RNA (sgRNA) assembles with Cas9 endonuclease to constitute a two-component surveillance complex, also termed Cascade (CRISPR-associated complex for antiviral defense) $)^{31}$. In the interference step, each complex searches for the nucleic acid sequence that matches its crRNA. Stable binding to the target DNA sequence requires the presence of a protospacer adjacent motif (PAM) that resides three bases downstream of the sgRNA recognition $\operatorname{site}^{30}$. As the surveillance complex scans along a DNA sequence, its Cas protein binds specifically to the PAM sequence and unwinds the double-stranded DNA, making the opened DNA spatially accessible for hybridizations with the crRNA ${ }^{32}$. Cas9's HNH and RuvC endonuclease domains cleave the complementary and non-complementary strand to the crRNA respectively, creating blunt DSB at the targeted locus ${ }^{33}$. For type II CRISPR/Cas system, accurate sequence recognition is realized by high-affinity Watson-Crick base pairing, leading to target Cas9-mediated interference and adaptive immunity to future invasions ${ }^{34,35}$. 

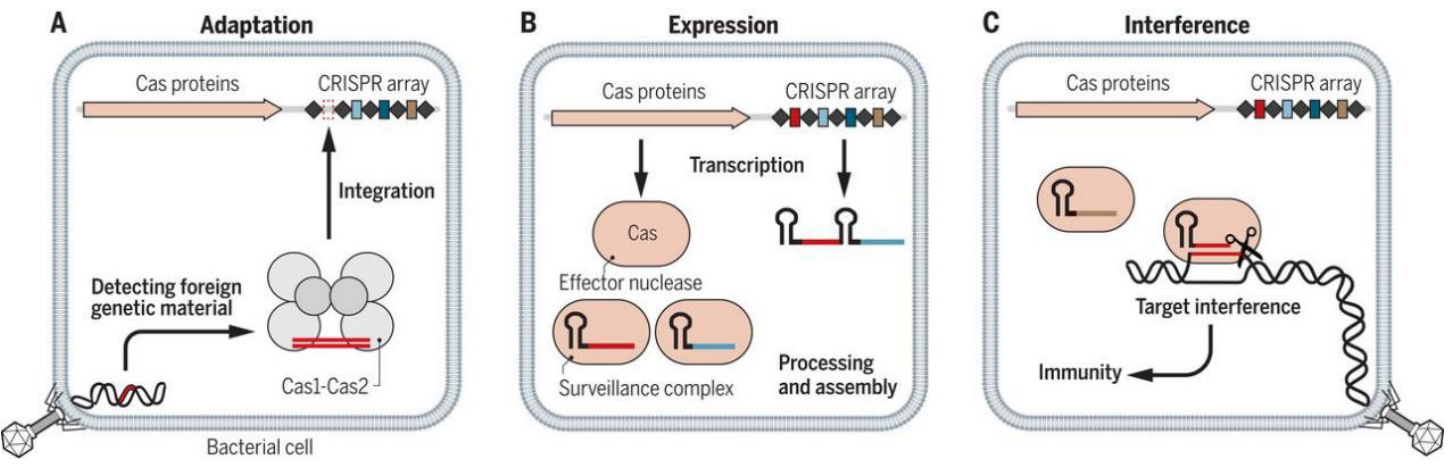

Figure 2: Acquisition of Adaptive Immunity via CRISPR/Cas System. (A) Foreign genetic material that invades the bacterial host cell will be first captured by the Cas1-Cas2 complex and then integrated into the CRISPR array. The diamond motifs in the CRISPR array represent the repeat regions. The square motifs represent the spacer regions, that contain the invading genetic elements. (B) Cas gene, CRISPR array, and tracrRNA gene (not shown in figure 2) will be transcribed into Cas effector nuclease, crRNA, and tracrRNA, respectively. crRNA and tracrRNA will fuse into a chimeric single-guide RNA (sgRNA) which will couple to a Cas9 nuclease and form a two-component surveillance complex. (C) Each surveillance complex will target the specific sequence that is complementary to its crRNA. Accurate recognition of foreign genetic material is based on Watson-Crick base pairing, resulting in target interference and immunity to future invasions. ${ }^{5}$

\section{Comparative analysis of genome editing mechanisms}

In recent years, a large number of genome projects have been implemented in search of some novel, operable, and affordable technologies that facilitate the exploration of specific gene function as well as its associated phenotype. Efficient genome editing not only provides a more comprehensive view of the basic science but also conveys great implications in the clinical, pharmacological, and biotechnical realms. Popular gene editing technologies use chimeric programmable nucleases to induce double-strand breaks (DSBs) which stimulate subsequent site-specific genome alterations via two endogenous DNA repair mechanisms (Fig. 3). To achieve efficient genome editing, there must be a reliable system that can produce DSB at any genomic locus of interest. Common strategies used are zinc finger nuclease (ZFN), transcription activator-like effector nuclease (TALEN), and CRISPR (clustered regularly interspaced short palindromic repeats)/Cas (CRISPR associated nuclease) system. DSBs generated by these programmable nucleases can lead to different kinds of genetic modifications, including point mutation, gene insertions, gene deletions, and chromosomal rearrangements ${ }^{36}$. A series of studies have shown that introductions of DSBs can greatly increase the frequency of endogenous DNA repair mechanisms at the break site $^{6,37}$. Common cellular repair mechanisms include homology-directed recombination (HDR) and non-homologous end joining (NHEJ).

Both ZFN and TALEN are constructed by fusing a non-sequence-specific FokI cleavage module with a series of sequence-specific DNA binding module ${ }^{38}$, which are derived from zinc finger protein (ZFP) or transcription-activator like effector (TALE) protein. ZFN and TALEN work as heterodimers because the FokI nuclease domain requires dimerization to initiate cleavage with high gene-editing specificity ${ }^{36}$. These two techniques demonstrate a certain degree of versatility due to the programmability of the DNA binding portion ${ }^{26}$. Despite the viability, there are several limitations to the engineering of modular DNA-binding proteins. ZFP binding modules exhibit context-dependent binding specificity due to crosstalk between modules when assembled within a larger system ${ }^{16,17}$. Though the single-base DNA-binding TALE protein provides more design flexibility than the triplet-confined $\mathrm{ZFP}^{26}$, its gene editing efficiency is also restricted by the genetic environments ${ }^{15}$. For both techniques, the major concerns are the specificities of the DNA-binding domain and the nuclease domain as well as the associated off-target toxicity ${ }^{39}$. Besides, the engineering of new ZFN or TALEN involves some extensive screening processes, that are often labor-intensive and costly ${ }^{26}$.

Given the difficulties associated with ZFN and TALEN, researchers have long pursued a more versatile and viable technique for gene editing and regulation. Now, the most rapidly developing genome editing technology utilizes an RNA-guided programmable endonuclease, termed Cas9, derived from the type II CRISPR system in the microbial adaptive immune system $^{19,21,22,35}$. Snippets of an extrachromosomal element, called "protospacers", will be integrated into the host chromosome at the proximal end of the CRISPR array and transcribed into CRISPR RNA (crRNA). Short crRNA can base pair with complementary protospacer sequences of foreign viral targets ${ }^{22}$. The palindromic repeats of host DNA are transcribed into trans-activating CRISPR RNA (tracrRNA). Hybridization of mature crRNA to tracrRNA results in the formation of a chimeric single-guide RNA (sgRNA) that can direct the Cas9 nuclease to induce sequence-specific DNA cleavage and silencing of pathogenic DNA ${ }^{26}$.

Recent genomic and biochemical studies have shown that a "seed sequence" of RNA nucleotides within the crRNA is particularly important for target recognition and cleavage specificity ${ }^{40,41}$. In type II CRISPR/Cas system, the seed sequence is defined by a 
GG-dinucleotide-containing protospacer adjacent motif (PAM) upstream of the crRNA-binding site on the DNA target $^{22,26,41}$. PAM determines the target search and interference mechanisms ${ }^{42}$ of Cas9, facilitating self versus non-self discrimination ${ }^{43}$ by the CRISPR system. Computational studies have shown that the delivery of multiple Cas9 endonucleases with difference PAM enables orthogonal genome engineering, where simultaneous and independent gene manipulations can be applied at multiple loci in bacterial and human cells ${ }^{44,45}$. Site-specific cleavage depends on both Watson-Crick base pairing complementarity between crRNA and the target protospacer DNA, and the presence of PAM adjacent to the complementary region on the target

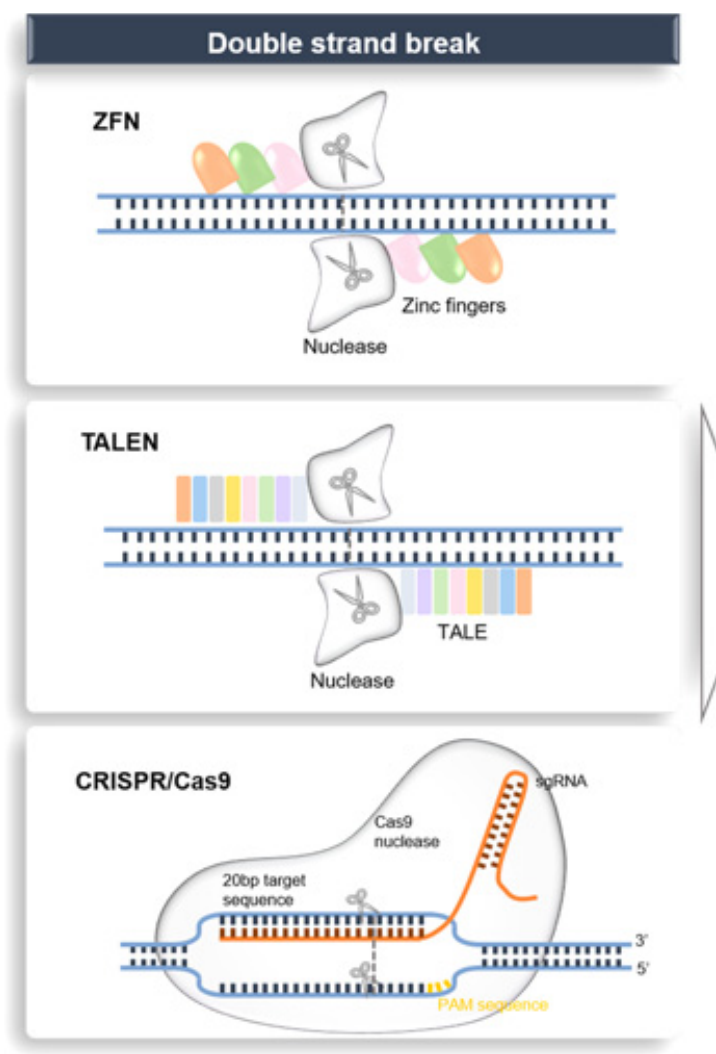

$\mathrm{DNA}^{40}$. DNA cleavage is performed by Cas9 endonuclease: it contains two catalytic domains $\mathrm{HNH}$ and RuvC, in which $\mathrm{HNH}$ is named after the histidine-asparagine-histidine residues in the activity site, and RuvC is termed for an E. coli protein involved in DNA repair ${ }^{46,47}$. Upon binding to the PAM sequence, Cas9 unwinds the double-stranded DNA, allowing the $\mathrm{HNH}$ domain to cut at sites complementary to the crRNA-guide sequence. Respectively, the RuvC domain will cleave the non-complementary strand. sgRNA-mediated DNA cleavage at the specific genomic locus generates a blunt-ended DSB for subsequent DNA repair via HDR or NHEJ.

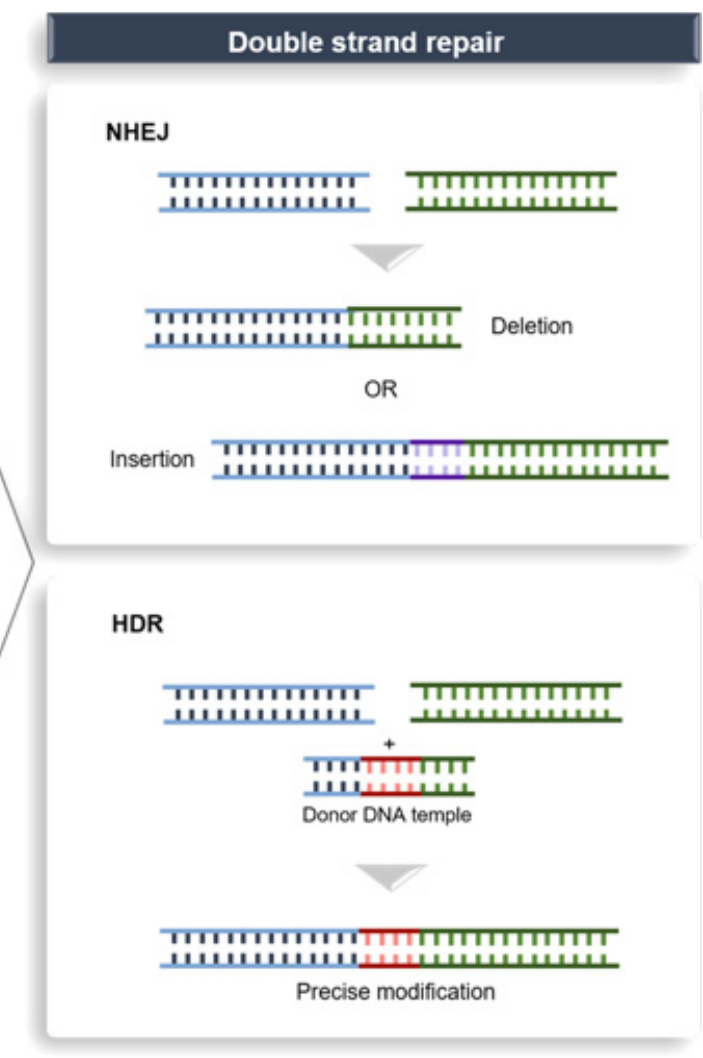

Figure 3: Technologies Utilize DNA Repair Mechanisms for Gene Editing. (Double-strand break; left) Early gene editing strategies include zing finger nuclease (ZFN) and transcription activator-like effector nuclease (TALEN). Both are synthetic proteins consisted of one FokI nuclease domain fused with a series of DNA binding domains. Each DNA binding domain of ZFN and TALEN recognizes $3 \mathrm{bps}$ and $1 \mathrm{bp}$ of DNA respectively. Dimerization of FokI nuclease upon protein-guided DNA recognition results in cleavage and formation of double-strand breaks (DSBs). CRISPR/Cas system utilizes a simpler sgRNA-guided mechanism to produce site-specific DNA cleavage. Stable binding to the target DNA requires the presence of a protospacer adjacent motif (PAM) upstream of the sgRNA recognition site. After binding, HNH and RuvC endonuclease domains on Cas9 will cleave the complementary and non-complementary strands of target DNA respectively. This also results in the formation of DSB at the target locus. (Double-strand repair; right) Induced DSB greatly increases the frequency of gene modification at the target site via homology-directed recombination (HDR) or non-homologous end joining (NHEJ). HDR can achieve precise gene editing if an exogenous donor template is provided. The error-prone NHEJ pathway often produces frameshift mutations, including insertion and deletion (indel), at the targeted site that disrupt normal gene function. ${ }^{2}$

\section{Application of CRISPR/Cas9 in HIVIAIDS treatment}

In recent years, the CRISPR/Cas system has been gradually applied to the field of viral infectious diseases. It has been studied extensively for its antiviral utility to provide new and effective treatments for human immunodeficiency virus (HIV) infection, which eventually leads to Acquired Immune Deficiency Syndrome (AIDS) that are often fatal. Nowadays, HIV/AIDS persists as a major public health problem. Approximately 40 million people across the globe have been contracted with HIV-1, with an annual growth of new infections exceeds 2 million individuals worldwide. Highly active antiretroviral therapy (HAART), as the traditional treatment for AIDS, has contributed to a 
palpable decrease in morbidity and mortality among patients with advanced or late-stage $\operatorname{AIDS}^{48,49}$. Yet, HAART fails to eradicate latent viral reservoir in HIV-1/AIDS patients ${ }^{50,51}$. In latently-infected CD4+ T cells, reverse transcriptase converts HIV-1 into an integrated retroviral DNA, also termed provirus, that enters a dormant state to avoid host immune response ${ }^{52}$; dormant proviruses can be reactivated to generate replication-competent virus upon activation of $\mathrm{T}$ cells, leading to a rapid rebound of viral load upon interruption of HAART ${ }^{51,53-56}$

\subsection{Inhibitions of HIV-1 infections via Cas/sgRNA-mediated LTR disruptions}

Strategies that specifically target HIV-1 proviral DNA through excision, silencing, or elimination of gene elements are the potential solutions to the current limitation of HARRT. Early work by Ebina et al. in 2013 utilizes CRISPR/Cas9 gene modulation technology to successfully remove proviral DNA by targeting the long terminal repeat (LTR) regions ${ }^{57}$. Long terminal repeat (LTR) is an indispensable promoter sequence for HIV-1 nucleic acid integration and gene expression ${ }^{57}$. A significant reductions in LTR-driven expression and intracellular proviral load are observed as LTR-targeting CRISPR/Cas9 systems are transfected into the dormant HIV-1-infected cells ${ }^{57}$. Further studies by the Liao, Rodriguez, and O'Neil's labs examine the efficiencies of Cas9-mediated disruptions on non-coding LTR sequence in latently infected human cells at different stages of the viral lifecycle. Results from these studies demonstrate the antiviral effectiveness of CRISPR/Cas9 system in lowering HIV-1 viral expression via LTR eradication in both the pre-integration stage and the proviral stage $\mathrm{e}^{58-61}$. Cells pre-treated with sgRNAs and the CRISPR/Cas system can successfully target non-integrating HIV-1 virus, inhibit the integration of viral cDNA into the host genome, and thus render the infected cells resistance to HIV-1 infections ${ }^{57}$. Lentivirus-delivered CRISPR/Cas9 system is also engineered and utilized to induce excisions of LTR sequences from all examined tissues and circulating lymphocytes ${ }^{62}$; targeting conserved sites within LTR sequence results in drastic diminutions of HIV-1 replication and viral gene expression in a latently infected CD4+ T cell line ${ }^{56,63,64}$.

\subsection{Prevention of HIV-1 cellular entry by targeting host dependency factors}

In 2015, Research results published by the Liao lab demonstrate that targeting multiple sites of the HIV-1 genome could increase the efficiencies of viral genome elimination and viral lifecycle disruption ${ }^{59}$. Apart from the excision or deactivation of LTR via mutagenic disruption, CRISPR/Cas9 system can also prevent HIV-1 cellular entry by directly act on cell surface receptors, such as CD4+ receptor and CCR5/CXCR4 coreceptors ${ }^{65}$. These receptors, upon binding of HIV-1, allow attachment and penetration of virus into the host cell ${ }^{66}$. Early study reveals that $\mathrm{CD} 4+$ receptors and $\mathrm{CXCR} 4$ coreceptors are less suitable for targeting disruptions since they are vital for the proper functioning of immune system ${ }^{67}$; therefore, CCR5 receptors becomes the focus of many investigations.

According to experimental results, individuals carrying a homozygous 32 bps deletion of the CCR5 gene $(C C R 5 \Delta 32)$ naturally resist HIV-1 infections ${ }^{68}$. Researches conducted by Hutter et al. and Allers et al. examine the impact of CCR5A32 stem cell transplantation on $\mathrm{HIV}$-infected patient and report successful inhibition of both viral replication and rebound ${ }^{69}$. Immune reconstitution of $\mathrm{CD} 4+\mathrm{T}$ cells via CCR5432 stem cell transplantation effectively reduces CCR5 surface expression and prompts the replacement of long-lived host smatic cells with donor-derived HIV-resisting CD4+ T cells ${ }^{69,70}$. More studies conclude that disruptive mutations of the CCR5 gene enable a long-lasting resistance to HIV-1 invasion without perturbing the proper functioning and survival of host immune cells ${ }^{68,71,72}$. However, due to the lack of natural homozygous CCR5 432 donors, gene editing technologies are exploited to induce the desired mutation on CCR5 gene to facilitate HIV-1/AIDS gene therapy ${ }^{73}$. Previous studies have reported successful gene editing of CCR5 gene by ZFNs in vivo ${ }^{74,75}$. In 2017, the Xu lab utilizes CRISPR/Cas9 system to target CCR5 gene in human hematopoietic stem cells (HPSCs) and achieves long-term immunization from HIV-1 infection in vivo ${ }^{76}$. Silencing of CCR5 surface expression via CRISPR/Cas9 is proven to be stable and long-lasting, thus presenting a possible functional cure for HIV/AIDS in future clinical settings ${ }^{76}$.

\subsection{Limitations of using CRISPR/Cas9 system for HIVIAIDS treatment}

Indeed, CRISPR/Cas9 system has demonstrated its potential as a new therapeutic platform for HIV/AIDS intervention after the conventional HAART treatment. Nonetheless, more investigations are still needed to ensure the system's stability and adaptability for in vivo, in vitro, and, more recently, ex vivo gene therapies ${ }^{65,77}$. By far, the major issues regarding the clinical treatment of HIV/AIDS via CRISPR/Cas are the safety, specificity, and technical capability regarding the delivery of Cas9-expression vector into the host cell ${ }^{78,79}$. Even though high frequencies of CCR5 disruption can be achieved, transduction of CRISPR/Cas system via lentiviral vectors often result in toxicities and off-target events in primary $\mathrm{T}$ cells ${ }^{80,81}$. Therefore, despite that CRISPR/Cas technology offers hope for the eradication of HIV/AIDS, it still has many limitations as a new therapeutic. The application of CRISPR/Cas9 system to HIV treatment in real-world clinical settings still has a long way to go. 


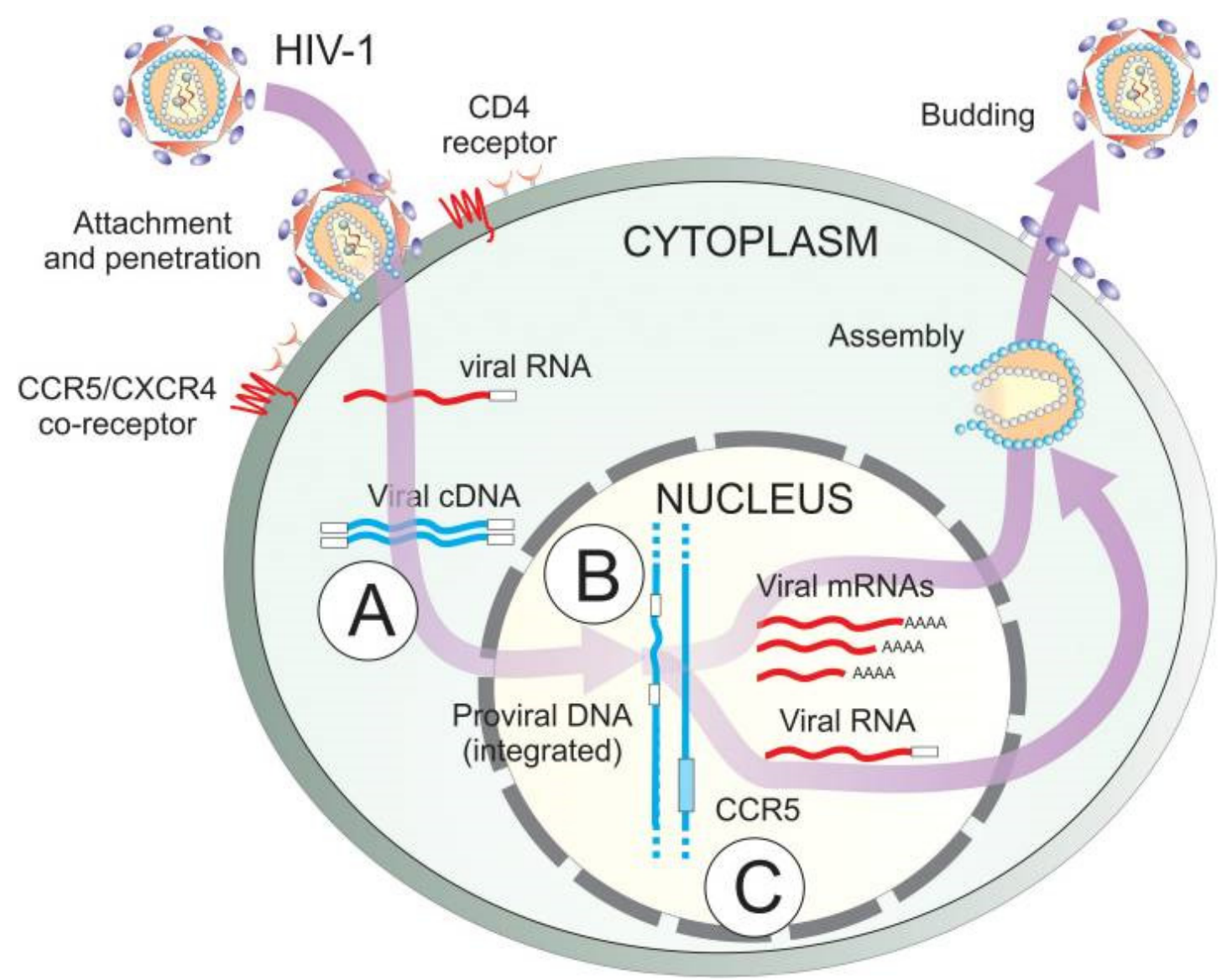

Figure 4: Different CRISPR/Cas9 therapeutic approaches against HIV infection based on viral life cycle. (viral life cycle) The first step of the viral life cycle involves the attachment and penetration of HIV-1 through interactions with cell surface receptor proteins, such as CD4+ receptor and CCR5/CXCR3 co-receptors. As the viral particle enters the intercellular environment, reverse transcriptase converts viral RNA into viral cDNA. cDNA is integrated into the host genome, and this proviral DNA serves as a template for the viral transcriptions and the subsequent assembly of virions that bud off to infect other host cells. (CRISPR/Cas9 intervention points) Nuclease-directed disruption can occur at the following three stages to inhibit HIV infection: (A) prevention of viral cDNA integration into the host genome via Cas9-mediated LTR disruption (B) targeting excision of integrated proviral DNA from host genome via Cas9-mediated LTR disruptions (C) inhibition of HIV cellular entry by targeting host dependency factors, such as CD4+ receptor and CCR5/CXCR4 coreceptor. ${ }^{65}$

\section{Conclusion and Future Prospect}

CRISPR/Cas-based technology has become one of the most versatile, adaptable, and efficient platform for genome engineering. It provides an accessible tool to regulate, modify, and visualize genomic elements and has now expanded to a broader range of organisms and cell types. As a revolutionary gene editing technology, the CRISPR/Cas system is used not only to build animal models, but also to correct defective disease-causing genes, eliminate viral infections, and treat different types of cancers. At present, researchers have been able to successfully exploit the CRISPR/Cas system to establish disease models in search of functions of specific genes and their associated phenotypes. However, though it is a promising technology, there are still several limitations that demand further investigations. Since genome manipulations produce permanent modifications, target specificity of CRISPR/Cas system is the most important issue, especially regarding to its clinical applications in treating human diseases. The effect of off-target toxicity is another restriction to the widespread implementation of CRISPR/Cas9 technology. The molecular structure of the CRISPR/Cas9 system is relatively complex as well; currently, researchers have not yet obtained a comprehensive understanding of the entire system. Therefore, whether there is a mutant Cas9 protein, in nature, that demonstrates higher editing accuracy, efficiency, or effectiveness entails further explorations.

\section{REFERENCES}

1. Doudna, J. A. \& Charpentier, E. Genome editing. The new frontier of genome engineering with CRISPR-Cas9. Science 346, 1258096, doi:10.1126/science.1258096 (2014).

2. Li, H. et al. Applications of genome editing technology in the targeted therapy of human diseases: mechanisms, advances and prospects. Signal Transduct Target Ther 5, 1, doi:10.1038/s41392-019-0089-y (2020).

3. Rudin, N., Sugarman, E. \& Haber, J. E. Genetic and physical analysis of double-strand break repair and recombination in Saccharomyces cerevisiae. 
Genetics 122, 519-534 (1989).

4. Cannan, W. J. \& Pederson, D. S. Mechanisms and Consequences of Double-Strand DNA Break Formation in Chromatin. J Cell Physiol 231, 3-14, doi:10.1002/jcp.25048 (2016).

5. Hsu, P. D., Lander, E. S. \& Zhang, F. Development and applications of CRISPR-Cas9 for genome engineering. Cell 157, 1262-1278, doi:10.1016/j.cell.2014.05.010 (2014).

6. Bibikova, M. et al. Stimulation of homologous recombination through targeted cleavage by chimeric nucleases. Mol Cell Biol 21, 289-297, doi:10.1128/MCB.21.1.289-297.2001 (2001).

7. Haber, J. E. A Life Investigating Pathways That Repair Broken Chromosomes. Annu Rev Genet 50, 1-28, doi:10.1146/annurev-genet-120215-035043 (2016).

8. Yeh, C. D., Richardson, C. D. \& Corn, J. E. Advances in genome editing through control of DNA repair pathways. Nat Cell Biol 21, 1468-1478, doi:10.1038/s41556-019-0425-z (2019).

9. Lander, E. S. The Heroes of CRISPR. Cell 164, 18-28, doi:10.1016/j.cell.2015.12.041 (2016).

10. Urnov, F. D., Rebar, E. J., Holmes, M. C., Zhang, H. S. \& Gregory, P. D. Genome editing with engineered zinc finger nucleases. Nat Rev Genet 11, 636-646, doi:10.1038/nrg2842 (2010).

11. Buck-Koehntop, B. A. et al. Molecular basis for recognition of methylated and specific DNA sequences by the zinc finger protein Kaiso. Proc Natl Acad Sci U S A 109, 15229-15234, doi:10.1073/pnas.1213726109 (2012).

12. Carroll, D. Genome engineering with zinc-finger nucleases. Genetics $188, \quad 773-782$, doi:10.1534/genetics.111.131433 (2011).

13. Miller, J. C. et al. A TALE nuclease architecture for efficient genome editing. Nat Biotechnol 29, 143-148, doi:10.1038/nbt.1755 (2011).

14. Li, T. et al. TAL nucleases (TALNs): hybrid proteins composed of TAL effectors and FokI DNA-cleavage domain. Nucleic Acids Res 39, 359-372, doi:10.1093/nar/gkq704 (2011).

15. Juillerat, A. et al. Comprehensive analysis of the specificity of transcription activator-like effector nucleases. Nucleic Acids Res 42, 5390-5402, doi:10.1093/nar/gku155 (2014).

16. Sander, J. D. et al. Selection-free zinc-finger-nuclease engineering by context-dependent assembly (CoDA). Nat Methods $\mathbf{8}$, 67-69, doi:10.1038/nmeth.1542 (2011).

17. Maeder, M. L. et al. Rapid "open-source" engineering of customized zinc-finger nucleases for highly efficient gene modification. Mol Cell 31, 294-301, doi:10.1016/j.molcel.2008.06.016 (2008).

18. Ishino, Y., Shinagawa, H., Makino, K., Amemura, M. \& Nakata, A. Nucleotide sequence of the iap gene, responsible for alkaline phosphatase isozyme conversion in Escherichia coli, and identification of the gene product. $J$ Bacteriol 169, 5429-5433, doi:10.1128/jb.169.12.5429-5433.1987 (1987).

19. Mojica, F. J., Diez-Villasenor, C., Soria, E. \& Juez, G. Biological significance of a family of regularly spaced repeats in the genomes of Archaea, Bacteria and mitochondria. Mol Microbiol 36, 244-246, doi:10.1046/j.1365-2958.2000.01838.x (2000).

20. Kunin, V., Sorek, R. \& Hugenholtz, P. Evolutionary conservation of sequence and secondary structures in CRISPR repeats. Genome Biol 8, R61, doi:10.1186/gb-2007-8-4-r61 (2007).

21. Jansen, R., Embden, J. D., Gaastra, W. \& Schouls, L. M. Identification of genes that are associated with DNA repeats in prokaryotes. Mol Microbiol 43, 1565-1575, doi:10.1046/j.1365-2958.2002.02839.x (2002).

22. Jinek, M. et al. A programmable dual-RNA-guided DNA endonuclease in adaptive bacterial immunity. Science 337, 816-821, doi:10.1126/science.1225829 (2012).

23. Bolotin, A., Quinquis, B., Sorokin, A. \& Ehrlich, S. D. Clustered regularly interspaced short palindrome repeats (CRISPRs) have spacers of extrachromosomal origin. Microbiology 151, 2551-2561, doi:10.1099/mic.0.28048-0 (2005).

24. Barrangou, R. \& Marraffini, L. A. CRISPR-Cas systems: Prokaryotes upgrade to adaptive immunity. Mol Cell 54, 234-244, doi:10.1016/j.molcel.2014.03.011 (2014).

25. Mali, P. et al. RNA-guided human genome engineering via Cas9. Science 339, 823-826, doi:10.1126/science.1232033 (2013).

26. Gaj, T., Gersbach, C. A. \& Barbas, C. F., 3rd. ZFN, TALEN, and CRISPR/Cas-based methods for genome engineering. Trends Biotechnol 31, 397-405, doi:10.1016/j.tibtech.2013.04.004 (2013).

27. Jiang, W., Bikard, D., Cox, D., Zhang, F. \& Marraffini, L. A. RNA-guided editing of bacterial genomes using CRISPR-Cas systems. Nat Biotechnol 31, 233-239, doi:10.1038/nbt.2508 (2013).

28. Nunez, J. K. et al. Cas1-Cas2 complex formation mediates spacer acquisition during CRISPR-Cas adaptive immunity. Nat Struct Mol Biol 21, 528-534, doi:10.1038/nsmb.2820 (2014).

29. Chylinski, K., Le Rhun, A. \& Charpentier, E. The tracrRNA and Cas9 families of type II CRISPR-Cas immunity systems. RNA Biol 10, 726-737, doi:10.4161/rna.24321 (2013).

30. Karvelis, T. et al. crRNA and tracrRNA guide Cas9-mediated DNA interference in Streptococcus thermophilus. RNA Biol 10, 841-851, doi:10.4161/rna.24203 (2013).

31. Wiedenheft, B. et al. Structures of the RNA-guided surveillance complex from a bacterial immune system. Nature 477, 486-489, doi:10.1038/nature10402 (2011).

32. Gleditzsch, D. et al. PAM identification by 
CRISPR-Cas effector complexes: diversified mechanisms and structures. RNA Biol 16, 504-517, doi:10.1080/15476286.2018.1504546 (2019).

33. Gasiunas, G., Barrangou, R., Horvath, P. \& Siksnys, V. Cas9-crRNA ribonucleoprotein complex mediates specific DNA cleavage for adaptive immunity in bacteria. Proc Natl Acad Sci U S A 109, E2579-2586, doi:10.1073/pnas.1208507109 (2012).

34. Knott, G. J. \& Doudna, J. A. CRISPR-Cas guides the future of genetic engineering. Science 361, 866-869, doi:10.1126/science.aat5011 (2018).

35. Terns, M. P. \& Terns, R. M. CRISPR-based adaptive immune systems. Curr Opin Microbiol 14, 321-327, doi:10.1016/j.mib.2011.03.005 (2011).

36. Song, M., Kim, Y. H., Kim, J. S. \& Kim, H. Genome engineering in human cells. Methods Enzymol 546, 93-118, doi:10.1016/B978-0-12-801185-0.00005-2 (2014).

37. Jasin, M. \& Rothstein, R. Repair of strand breaks by homologous recombination. Cold Spring Harb Perspect Biol 5, a012740, doi:10.1101/cshperspect.a012740 (2013).

38. Kim, Y. G., Cha, J. \& Chandrasegaran, S. Hybrid restriction enzymes: zinc finger fusions to Fok I cleavage domain. Proc Natl Acad Sci U S A 93, 1156-1160, doi:10.1073/pnas.93.3.1156 (1996).

39. Szczepek, M. et al. Structure-based redesign of the dimerization interface reduces the toxicity of zinc-finger nucleases. Nat Biotechnol 25, 786-793, doi:10.1038/nbt1317 (2007).

40. Jiang, F. \& Doudna, J. A. CRISPR-Cas9 Structures and Mechanisms. Annu Rev Biophys 46, 505-529, doi:10.1146/annurev-biophys-062215-010822 (2017).

41. Wiedenheft, B. et al. RNA-guided complex from a bacterial immune system enhances target recognition through seed sequence interactions. Proc Natl Acad Sci U S A 108, 10092-10097, doi:10.1073/pnas.1102716108 (2011).

42. Mojica, F. J. M., Diez-Villasenor, C., Garcia-Martinez, J. \& Almendros, C. Short motif sequences determine the targets of the prokaryotic CRISPR defence system. Microbiology 155, 733-740, doi:10.1099/mic.0.023960-0 (2009).

43. Marraffini, L. A. \& Sontheimer, E. J. Self versus non-self discrimination during CRISPR RNA-directed immunity. Nature 463, 568-571, doi:10.1038/nature08703 (2010).

44. Esvelt, K. M. et al. Orthogonal Cas9 proteins for RNA-guided gene regulation and editing. Nat Methods 10, 1116-1121, doi:10.1038/nmeth.2681 (2013).

45. Fonfara, I. et al. Phylogeny of Cas 9 determines functional exchangeability of dual-RNA and Cas9 among orthologous type II CRISPR-Cas systems. Nucleic Acids Res 42, 2577-2590, doi:10.1093/nar/gkt1074 (2014).

46. Jinek, M. et al. Structures of Cas9 endonucleases reveal RNA-mediated conformational activation. Science 343, 1247997, doi:10.1126/science. 1247997 (2014).

47. Nishimasu, H. et al. Crystal structure of Cas9 in complex with guide RNA and target DNA. Cell 156, 935-949, doi:10.1016/j.cell.2014.02.001 (2014).

48. Palella, F. J., Jr. et al. Declining morbidity and mortality among patients with advanced human immunodeficiency virus infection. HIV Outpatient Study Investigators. $N$ Engl $J$ Med 338, 853-860, doi:10.1056/NEJM199803263381301 (1998).

49. Gandhi, R. T. et al. The effect of raltegravir intensification on low-level residual viremia in HIV-infected patients on antiretroviral therapy: a randomized controlled trial. PLoS Med 7, doi:10.1371/journal.pmed.1000321 (2010).

50. Finzi, D. et al. Identification of a reservoir for HIV-1 in patients on highly active antiretroviral therapy. Science 278, $1295-1300$ doi:10.1126/science.278.5341.1295 (1997).

51. Chun, T. W. et al. Quantification of latent tissue reservoirs and total body viral load in HIV-1 infection. Nature 387, 183-188, doi:10.1038/387183a0 (1997).

52. Mougel, M., Houzet, L. \& Darlix, J. L. When is it time for reverse transcription to start and go? Retrovirology 6, 24, doi:10.1186/1742-4690-6-24 (2009).

53. Wong, J. K. et al. Recovery of replication-competent HIV despite prolonged suppression of plasma viremia. Science 278, 1291-1295, doi:10.1126/science.278.5341.1291 (1997).

54. Siliciano, J. D. et al. Long-term follow-up studies confirm the stability of the latent reservoir for HIV-1 in resting CD4+ T cells. Nat Med 9, 727-728, doi: $10.1038 / \mathrm{nm} 880$ (2003).

55. Hermankova, M. et al. Analysis of human immunodeficiency virus type 1 gene expression in latently infected resting $\mathrm{CD} 4+\mathrm{T}$ lymphocytes in vivo. $J$ Virol 77, 7383-7392, doi:10.1128/jvi.77.13.7383-7392.2003 (2003).

56. Kaminski, R. et al. Elimination of HIV-1 Genomes from Human T-lymphoid Cells by CRISPR/Cas9 Gene Editing. Sci Rep 6, 22555, doi:10.1038/srep22555 (2016).

57. Ebina, H., Misawa, N., Kanemura, Y. \& Koyanagi, Y. Harnessing the CRISPR/Cas9 system to disrupt latent HIV-1 provirus. Sci Rep 3, 2510, doi:10.1038/srep02510 (2013).

58. Craigie, R. \& Bushman, F. D. HIV DNA integration. Cold Spring Harb Perspect Med 2, a006890, doi:10.1101/cshperspect.a006890 (2012).

59. Liao, H. K. et al. Use of the CRISPR/Cas9 system as an intracellular defense against HIV-1 infection in human cells. Nat Commun 6, 6413, doi:10.1038/ncomms7413 (2015).

60. O'Neil, P. K. et al. Mutational analysis of HIV-1 long terminal repeats to explore the relative contribution 
of reverse transcriptase and RNA polymerase II to viral mutagenesis. J Biol Chem 277, 38053-38061, doi:10.1074/jbc.M204774200 (2002).

61. Rodriguez, M. A. et al. Genetic and functional characterization of the LTR of HIV-1 subtypes A and $\mathrm{C}$ circulating in India. AIDS Res Hum Retroviruses 23, 1428-1433, doi:10.1089/aid.2007.0152 (2007).

62. $\mathrm{Hu}, \mathrm{W}$. et al. RNA-directed gene editing specifically eradicates latent and prevents new HIV-1 infection. Proc Natl Acad Sci U S A 111, 11461-11466, doi:10.1073/pnas.1405186111 (2014).

63. Kaminski, R. et al. Excision of HIV-1 DNA by gene editing: a proof-of-concept in vivo study. Gene Ther 23, 690-695, doi:10.1038/gt.2016.41 (2016).

64. Soriano, V. Hot News: Gene Therapy with CRISPR/Cas9 Coming to Age for HIV Cure. AIDS Rev 19, 167-172 (2017).

65. Saayman, S., Ali, S. A., Morris, K. V. \& Weinberg, M. S. The therapeutic application of CRISPR/Cas9 technologies for HIV. Expert Opin Biol Ther 15, 819-830, doi:10.1517/14712598.2015.1036736 (2015).

66. Bleul, C. C., Wu, L., Hoxie, J. A., Springer, T. A. \& Mackay, C. R. The HIV coreceptors CXCR4 and CCR5 are differentially expressed and regulated on human T lymphocytes. Proc Natl Acad Sci U S A 94, 1925-1930, doi:10.1073/pnas.94.5.1925 (1997).

67. Nagasawa, T. et al. Defects of B-cell lymphopoiesis and bone-marrow myelopoiesis in mice lacking the CXC chemokine PBSF/SDF-1. Nature 382, 635-638, doi:10.1038/382635a0 (1996).

68. Samson, M. et al. Resistance to HIV-1 infection in caucasian individuals bearing mutant alleles of the CCR-5 chemokine receptor gene. Nature 382, 722-725, doi:10.1038/382722a0 (1996).

69. Allers, K. et al. Evidence for the cure of HIV infection by CCR5Delta32/Delta32 stem cell transplantation. Blood 117, 2791-2799, doi:10.1182/blood-2010-09-309591 (2011).

70. Hutter, G. et al. Long-term control of HIV by CCR5 Delta32/Delta32 stem-cell transplantation. $N$ Engl $J$ Med 360, 692-698, doi:10.1056/NEJMoa0802905 (2009).

71. Biti, R. et al. HIV-1 infection in an individual homozygous for the CCR5 deletion allele. Nat Med 3, 252-253, doi:10.1038/nm0397-252 (1997).
72. Cho, S. W., Kim, S., Kim, J. M. \& Kim, J. S. Targeted genome engineering in human cells with the Cas9 RNA-guided endonuclease. Nat Biotechnol 31, 230-232, doi:10.1038/nbt.2507 (2013).

73. Zimmerman, P. A. et al. Inherited resistance to HIV-1 conferred by an inactivating mutation in CC chemokine receptor 5: studies in populations with contrasting clinical phenotypes, defined racial background, and quantified risk. Mol Med 3, 23-36 (1997).

74. Holt, N. et al. Human hematopoietic stem/progenitor cells modified by zinc-finger nucleases targeted to CCR5 control HIV-1 in vivo. Nat Biotechnol 28, 839-847, doi:10.1038/nbt.1663 (2010).

75. Perez, E. E. et al. Establishment of HIV-1 resistance in CD4+ T cells by genome editing using zinc-finger nucleases. Nat Biotechnol 26, 808-816, doi:10.1038/nbt1410 (2008).

76. $\mathrm{Xu}, \mathrm{L}$. et al. CRISPR/Cas9-Mediated CCR5 Ablation in Human Hematopoietic Stem/Progenitor Cells Confers HIV-1 Resistance In Vivo. Mol Ther 25, 1782-1789, doi:10.1016/j.ymthe.2017.04.027 (2017).

77. Xiao, Q., Guo, D. \& Chen, S. Application of CRISPR/Cas9-Based Gene Editing in HIV-1/AIDS Therapy. Front Cell Infect Microbiol 9, 69, doi:10.3389/fcimb.2019.00069 (2019).

78. Lino, C. A., Harper, J. C., Carney, J. P. \& Timlin, J. A. Delivering CRISPR: a review of the challenges and approaches. Drug Deliv 25, 1234-1257, doi:10.1080/10717544.2018.1474964 (2018).

79. Xu, C. L., Ruan, M. Z. C., Mahajan, V. B. \& Tsang, S. H. Viral Delivery Systems for CRISPR. Viruses 11, doi:10.3390/v11010028 (2019).

80. Wang, W. et al. CCR5 gene disruption via lentiviral vectors expressing Cas 9 and single guided RNA renders cells resistant to HIV-1 infection. PLoS One 9, e115987, doi:10.1371/journal.pone.0115987 (2014).

81. Cradick, T. J., Fine, E. J., Antico, C. J. \& Bao, G. CRISPR/Cas9 systems targeting beta-globin and CCR5 genes have substantial off-target activity. Nucleic Acids Res 41, 9584-9592, doi:10.1093/nar/gkt714 (2013). 